\title{
Historein
}

Vol $2(2000)$

Heterodoxies: Constructions of Identities and Otherness in Medieval and Early Modern Europe

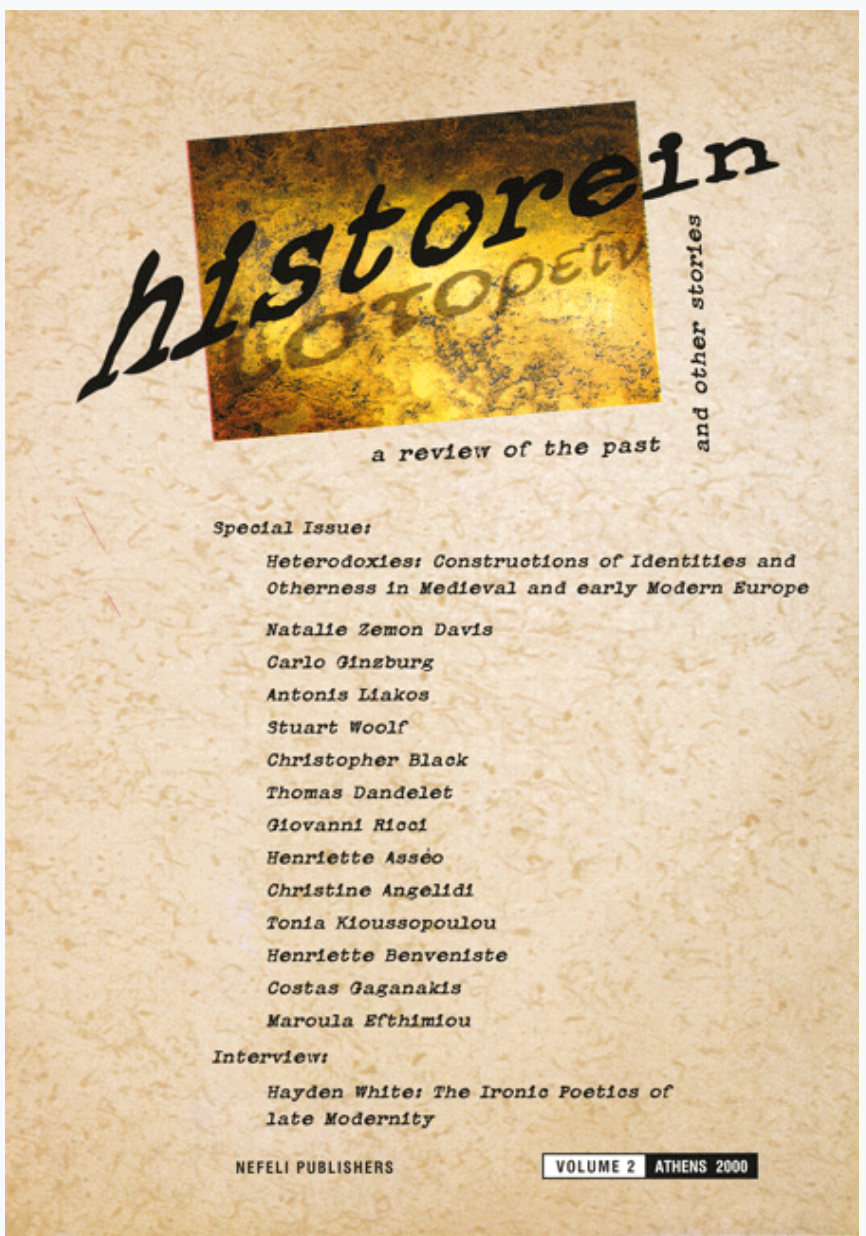

\section{Visibilité et identité flottante: Les 'Bohémiens' ou 'Egyptiens' (Tsiganes) dans la France de l'Ancien Régime}

Henriette Asséo

doi: $10.12681 /$ historein.116

Copyright @ 2012, Henriette Asséo

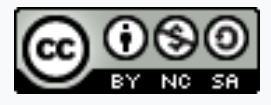

This work is licensed under a Creative Commons Attribution-NonCommercialShareAlike 4.0.

\section{To cite this article:}

Asséo, H. (2001). Visibilité et identité flottante: Les 'Bohémiens' ou 'Egyptiens' (Tsiganes) dans la France de l'Ancien Régime. Historein, 2, 109-122. https://doi.org/10.12681/historein.116 


\section{Visibilité \\ et identité}

flottante:

\section{les "Bohémiens}

ou Égyptiens"

(Tsiganes)

\section{dans la France}

\section{de l'Ancien}

Régime

Henriette Asséo
Nous voudrions montrer comment s'est effectuée la "construction identitaire", selon une formule démarquée du concept allemand de "construction confessionnelle", des "Bohémiens ou Égyptiens" - c'est ainsi que l'on appelait les Tsiganes - dans la France de l'Ancien Régime.

Les documents mentionnent, en effet, à partir de 1417, la venue de gens singuliers conduits par des "Ducs et comtes de Petite Égypte". Ces groupes, peu nombreux mais mobiles et fortement soudés, présentaient déjà ce mélange d'arrogance et de gueuserie que Jacques Callot a traduit, bien plus tard, dans une série de gravures sur la guerre de Trente Ans.

Mais, contrairement à l'opinion commune, ces Egyptiens reçurent de la part des villes - du moins jusqu'en 1480-1500 - l'accueil réservé à des personnages présentant des lettres de protection de l'Empereur, du roi de Bohême, des rois de France, des ducs de Savoie et de Bourgogne et même une habile copie de lettres papales. ${ }^{1}$ Ces lettres recommandaient les Bohémiens à toute la chrétienté comme "d'honnestes pélerins", expiant une apostasie temporaire. Ils auraient, disaient-ils, quitté une région à la localisation incertaine dite de "Gypte la Mineure" pour fuir l'Islam.

A partir du XVlème siècle, la mission des Tsiganes devint essentiellement militaire. Les "ducs et comtes de Petite Égypte", se métamorphosèrent en "capitaines" conducteurs de "mesnages égyptiens". Ces compagnies parcouraient le royaume en suivant les frontières mouvantes de l'État de guerre; elles proposaient aussi leurs services aux seigneurs indisciplinés, engagés dans les camps opposés des "troubles de religion" ou ralliés plus tard à l'agitation frondeuse. 
Cette ciculation entre guerres aux frontières et plat pays appellent deux sortes de remarques:

Des compagnies furent autorisées à circuler dans le royaume en dépit des ordonnances édictées par ces mêmes princes, François ler, Henri ll ou Henri IV, contre "les gens se disant Bohesmiens ou Egyptiens".

Le "mesnage d'Egyptiens" semble reposer sur une véritable structure familiale, difficile à mettre à jour par la nature des documents disponibles.

La morphologie des troupes bohémiennes leur conférait une puissante visibilité collective, les faisant glisser progressivement de la mobilité autorisée par l'argument religieux à la circulation redoutée d'une troupe armée et quelque peu prédatrice.

Les états successifs des compagnies des capitaines Lescuyer, en campagne tout au long du XVlème siècle, montrent bien cette évolution.

Un capitaine Anthoine Lescuier avait reçu une licence, le 18 février 1522, pour circuler dans le duché de Bretagne avec ses gens, en tant que "lieutenant du compte de Petite-Egypte." Cette autorisation avait été précédée d'un mandement de congé et licence du 31 mai 1508, "pour les egiciens estans à présent en ceste vill de nantes, de passer par le pays de Bretagne en allant au Mont-Saint-Michel, où ilz porront séjourner en faisant le voyage l'espace de vingt jours. ${ }^{3}$ Un itinéraire habituel de pélerinage par la route du Mont-Saint-Michel justifie les déplacements d'une compagnie militaire uniquement désignée par la qualification assez énigmatique de son chef.

Mais un document notarié éclaire la structure interne d'une compagnie semblable. Un acte trouvé dans les minutes de Claude de Baigneaulx, notaire à Chartres, du 17 mai 1571 nous met en présence d'un capitaine de bohémiens, Nicolas Lescuyer, et de son lieutenant, François Aubert, passant un contrat en bonne et due forme devant notaire. ${ }^{4}$ Nicolas Lescuyer se déclarait "natif de la Petite-Egypte", capitaine et conducteur de trois ménages de sa "compaignie", et il affirmait que François Aubert avait bien été sous sa charge avec son épouse, sa mère ainsi que "famille et serviteurs", et qu'il s'était toujours bien comporté selon le droit que le roi leur avait donné de "passer par tout le royaulme de France en bonne seureté avec lesdictz mesnages sans les molester en auculne chose". François Aubert demandait à quitter la troupe pour rejoindre ses frères avec lesquels il voulait former une autre compagnie. L'acte enregistrait le congé et certifiait la validité des lettres, passeports et sauf-conduits en possession des Egyptiens.

\section{La visibilité collective des "Bohémiens ou Egyptiens"}

Peut-on identifier plus avant la nature de l'inscription territoriale dans un espace national de ces compagnies qui se présentent délibérement comme des mercenaires? En d'autres termes, peut-on aller jusqu'à dire que les gens dits de "rasse égyptienne" qui se mirent au service des Princes ont pu former par le réseau étendu de leurs liens une forme cohérente d'entreprise familiale de guerre? 
Les interrogatoires du capitaine La Fleur, mené à Thionville en 1603, qui affirmait être un "Egiptien françois", en ce sens qu'il ne servait que le camp catholique, apporte quelques éclaircissement sur le comportement militaire des premières dynasties de capitaines de Bohémiens.

La Lorraine, convoitée depuis le partage de Charlemagne, se prêtait au service de Princes variés. Elle devint un foyer de formation et de distribution entre "Egiptiens francois" et "Egiptiens allemans" par le biais du service des armes. Car le partage entre Egyptiens français servant le camp catholique et Egyptiens allemands appartenant au camp protestant, s'est effectué au coup par coup en fonction des variations d'allégeance à l'égard des chefs militaires dont ils dépendaient. ${ }^{5}$

Ainsi, à Thionville, en mai 1603, le capitaine Jean de la Fleur fut longuement interrogé par Jehan de Wiltz, gouverneur, capitaine et prévôt de de cette ville et prévôté. ${ }^{6}$ Ce dossier est particulièrement instructif parce que le magistrat manifeste une curiosité véritable et qu'il joint au questionnaire nombre de pièces à l'appui parmi lesquelles des certificats et des passeports en bonne forme. ${ }^{7}$ Selon les recommandations des gouverneurs, des poursuites avaient été engagées à l'encontre de ce capitaine soupçonné d'un vol effectué pendant son service au camp de Bastogne. Arrêté quelques mois auparavant, il était parvenu à s'évader avec un autre jeune garçon et depuis il n'avait cessé de hanter le pays, "avecq ses deux mesnaiges, vivant et desrobant comme sont accoustumés de faire gens de sa race, et d'aultant qu'il est raisonnable que ung chacun face le debvoir de son office pour faire punir les delinquans en icelluy." Le capitaine la Fleur était donc capturé à Thionville avec un jeune garçon, qui serait, apparement, le fils d'un conducteur de ménage égyptien nommé Jean de la Verdure.

La liste des questions à poser jointe à la lettre explique la précision des interrogatoires. Le gouverneur décida d'éclaircir trois points: la signification réelle de l'appartenance à la "rasse des Egyptiens," la composition des ménages d'Egyptiens, la place de chacun et surtout, il chercha à reconstituer le périple militaire du capitaine afin de déterminer l'origine des biens en sa possession.

Jean de la Fleur accepta de répondre de bonne grâce aux questions. II affirme avec fierté son appartenance à la nation égyptienne et il expose sans détour: "estre de la Rasse d'Egyptiens mais natif de Rasié pays de Lorrayne et proche de St Mihiel où il a été baptisé à l'eglise catholique comme ung autre chrestien." Le magistrat ne s'arrête pas à cette surprenante déclaration; bien plus que la qualité d'étranger, l'appartenance à la "rasse d'égyptiens" semble impliquer pour lui une tendance fâcheuse à l'approximation dans la déclinaison des liens familiaux et en particulier des accommodements avec le mariage fortement pré-tridentins.

De fait, la condition matrimoniale du capitaine paraît fort imprécise. Servant aux Pays-Bas, il avait pris pour "garse" une femme mariée qu'il n'avait de ce fait pu épousé. Elle lui donna cinq enfants et il aimait à rappeler qu'ils étaient tous vivants, qu'il en avait mis deux aux écoles, tandis que les autres l'accompagnaient dans ses pérégrinations.

Cette union prolifique ne l'empêcha pas d'abandonner sa compagne, tombée malade près de 
Nivelle, et d'épouser en bonne et due forme une certaine Judith, rencontrée depuis. Le magistrat formulait aussitôt le soupçon de bigamie et lui demandait instamment s'il " $n$ 'a oncques heu deux femmes espousées en ung temps".

Mais les aventures conjugales du capitaine n'étaient pas terminées. Le magistrat l'interrogea sur le fait nettement plus grave de savoir s'il n'avait pas tué sa femme? Le capitaine admit avoir entendu parler d'une affaire passionnelle qui avait provoqué le meurtre d' un Egyptien. Alors qu'il logeait avec les autres Egyptiens à Stockeim, près d'Arlan, un certain Guillaume, l'un de ses compagnons, avait trouva sa femme "couché aupres d'ung aultre egiptien" et l'ombrageux bohémien les avait transpercés tous deux d'un coup d'épée. Or le capitaine avait pour habitude de confier en son absence à ce même Guillaume la conduite de la troupe. ${ }^{8}$

Comment le capitaine a-t-il commencé sa carrière? Fils de Julien et de Anne, tous deux originaires de Tilly-sur-Meuse en Lorraine, il fut, à l'âge de douze ans, placé comme laquais chez un gentilhomme Iorrain, le sieur d'Atrey. Son entrée dans la vie militaire se fit dans le régiment du comte de la Bourlotte (Borlotte), qu'il a servi pendant douze ans, puis sous les ordres du comte de Salm. II avait été aussi au régiment de Noircarme. A présent, il accompagnait deux ménages d'Egyptiens, eux aussi basés en Lorraine, et il s'apprêtait à rejoindre Monsieur de Châtel lui ayant promis d'être de ses troupes, "auquel effect il ait achepté ung cheval auprès du prevost d'Esch."

Ses propos reflétaient cette forme complexe de patriotisme professionnel propre aux armées du temps. II insista à plusieurs reprises sur sa fidélité au clan catholique qui l'avait conduit à toujours porter les armes pour le "service du Roy d'Espaigne et de leurs Altezes Sermes." II précisait "qu'il a toujours esté au service au Pays bas et en France avec feu le duc de Parme sans avoir esté oncques avecq l'ennemis ny hollandois." II parlait du service de son prince, I'Archiduc. II fut d'ailleurs fait prisonnier par les Hollandais aux alentours de Philippeville.

II mentionna aussi un curieux séjour en Hongrie où il avait été une fois "desoulz la charge du comte de Montecuculy." II dira ultérieurement avoir fait un voyage "parensemble en Hongrye" avec trois compagnons. Peut-être, faisait-il allusion à la période de la guerre de Quinze Ans quand le prince de Transylvanie, Sigismond Bathary, s'était rangé résolument aux côtés des Habsbourg? Ce conflit servit d'ailleurs de ban d'essai aux entrepreneurs de guerre.

Avec quels moyens vivait-il? Quand il était au service de quelque prince, il recevait une contribution de dix sols par jour: "comme ung aultre egiptien, lesquelz recepvant leur argent aucunes fois à Lion sur Ron ou a deniers pour eschange." Quand on lui posa à nouveau la question de savoir "s'il y a des vrays egiptiens," il fit la réponse suivante "dict qu'ils sont de la rase d'Egipte, mais né au pays de luxembourg et de Lorrayne." Mais là encore le magistrat ne cherche pas à faire préciser la nature exacte de la qualification de la "rasse d'Egyptiens"; elle est simplement pour lui la manière d'être, redoutable mais familière, de ces compagnies et par une 
remarquable tautologie, il considère que les Egyptiens sont "gens se disant Egyptiens," qualité que le capitaine ne renie pas.

Or cette réponse s'éclaire à la lumière d'un autre témoignage, celui de Jacques David, un jeune garçon de quinze ans interrogé le 2 mai 1603, en présence du baron de Wiltz. ${ }^{9}$ Son père se serait appelé Jehan de la Fontaine et il était de la bourgeoisie de Saint-Maixent. II aurait épousé une Egyptienne, nommée Marie. Durant la guerre de France, des "Egiptiens françoys" assassinèrent ses parents. Son frère parvint à s'enfuir mais lui-même fut fait prisonnier, "estant alhors encores bien jeusne et petit ilz ne luy firent rie." La raison du meurtre de ses parents venait, selon lui, du fait que "estiant gens de moyens ne voullant point aller permy les pays avecq eulx." II servait donc un Egyptien qui devait être ce Jean de la Verdure déjà mentionné par le capitaine La Fleur. S'il restait avec lui, c'était qu'il devait prendre soin "pour les assister à nourir" de ses jeunes frères et soeurs. Quand on l'interrogea sur des "voulleries" éventuelles, il eut cette réponse ironique que leur métier ou art ne valait plus rien car trop de gens, à présent, se mêlaient de "cestuy mestier, estant plus abille que luy."

Enfin le capitaine La Fleur expliquait comment la solde leur était délivré à Lyon et à Anvers. Le comte Lambert Philippe, Egyptien qualifié aussi de général, lui avait donné cette charge de capitaine au service des princes. Le comte Lambert Philippe tenait ses quartiers en HauteBourgogne et lui donnait des "lettres d'eschanges s'addressant au bancq de Lion ou de Venise ou bien à anvers pour recepvoir leurs gaiges ascavoir pour luy dix solz par jour et aultres de ses compaignions moeyennant qu'ilz soient filz chacun cincq solz par jour". Bien des embarras pour une solde journalière assez mince, mais on ne pourra pas accuser ce capitaine de n'avoir à sa disposition que des bribes d'une subculture de marginal.

Enfin, la plupart du temps, les femmes complétaient ces subsides en "disant la bonne aventure a d'aulcuns simples gens."

Ainsi la double architecture du monde tsigane de l'époque moderne transparaît à travers ce document: la fonction militaire qui ne fait pas différer la compagnie bohémienne des armées du temps, mais aussi l'existence de liens familiaux complexes, encore stabilités et pourtant essentiels à la construction identitaire.

Le repérage de la forme singulière de la compagnie de Bohémiens n'est que le préalable à notre démonstration. En effet elle laisse ouverte deux autres questions essentielles.

En premier lieu, il nous faudrait démontrer que la pérennité de la présence bohémienne $s^{\prime}$ inscrit dans une véritable reproduction généalogique, qu'il ne s'agit pas seulement d'une association temporaire de soldats mercenaires battant la campagne.

En second lieu il faudrait aussi montrer qu'il existe un véritable lien culturel ou biologique - peu importe - entre ces Bohémiens "d'en France" et la dispersion tsigane européenne. 
A première vue, une activité militaire, plus ou moins bien tolérée, devait assurer aux compagnies dotées d' une identité anthropologique d'ailleurs fort imprécise de "bohémiens ou Egyptiens" une existence collective pendant les temps troublés. Mais ceci ne permettrait pas de rendre compte de leur maintien physique, après la pacification définitive du royaume voulue par Louis XIV et ses administrateurs.

D'autant que Louis XIV et Colbert ont multiplié ordonnances et édits après 1662. La déclaration de juillet 1682 visait la dispersion de la compagnie bohémienne pourchassée pour "attroupements" et "oppressions." ${ }^{10}$ Cet objectif n'était pas nouveau mais le roi complétait le dispositif répressif en pourchassant les seigneurs qui donnaient retraite aux Bohémiens et surtout il menaçait explicitement de l'Hôpital l'Egyptienne qui courait la campagne en "faisant le mestier de bohémienne," disant la bonne aventure à un peuple crédule pour mieux commetre "larrecins et vouleries."

A partir des années 1700-1750, une postérité bien déclinante des compagnies tsiganes, si l'on songe à l'insolence affichée un siècle auparavant, circule encore dans le royaume.

Voici tout ce qu'il reste de la brillante compagnie de notre aimable capitaine La Fleur: une triste fin dans le bailliage de Vermandois, conclue par l'arrestation par le prévôt, le 30 juillet 1700, de Antoine Oudin, Suzanne de la Roche, Marie de la Tour, Marie de la Roche, Gabriel de La Fleur, Marie de la Roze et Marion de la Tour." Tous les inculpés, après interrogatoires sur la sellette, furent déclarés coupables; Antoine Oudin, "errant, vagabond et menant la vie de Bohesme" fut envoyé aux galères; les femmes furent rasées et bannies car de "qualitez et conditions bohémiennes et en faisant la profession depuis plusieurs années." Enfin, suivant les dispositions de la Déclaration royale de 1682, une enfant de cinq à six ans fut conduite à "I' hospitalle generalle de cette ville" pour y être élevée "comme les pauvres renfermez dans cette maison."

La menace des galères réservé aux hommes récidivistes a porté sur le devant de la scène paysanne des bohémiennes "errantes et vagabondes." La visibilité des bohémiens s'est donc progressivemnt déplacée: elle est passée de l'identification collective au repérage individuel, de la troupe picaresque d'hommes armés à la femme quémandeuse, noire de peau et d'âme, encombrée d'enfants aux yeux fureteurs. Selon sa déposition du 5 décembre 1728, un cavalier de maréchaussée de Tours à la résidence d'Amboise, fort satisfait de sa vigilance, n'a pas été long à saisir le manège d'une femme "estant sur le pont dans la ville à observer les passants, avons remarqué qu'une jeune femme de couleur bazanée, et d'une taille alegre avoit l'air d'une boyemienne". II en fait un portrait peu avenant mais immédiatement repérable : "le visage noir..., les yeux noirs, les sourcils aussi noirs, le visage long, les joues élevées, le nez gros, la bouche grande ayant de la barbe sur la lèvre supérieure, parlant bon francais, se donnant un air moqueur voulant faire l'agréable et cependant fort laide."12

En dépit de leur dénuement apparent, ces bohémiennes se sont révélées fort capables de tenir 
tête aux magistrats, sachant répondre avec pertinence en déclinant leur identité familiale sans rien nier de leur état de "bohémienne," tout en cachant du mieux l'exercice délictueux de la bonne aventure, le "mestier" qui sert à les identifier.

C'est donc la femme qui, par nécessité, a pris la relève d'une marginalité confirmée. La figure baroque de la Bohémienne exerçant son métier dans des campagnes de moins en moins accueillantes a connu une promotion mythique à la mesure du déclin social du groupe.

Est-ce à dire que la famille bohémienne a pour autant disparu? L' historien des sources judiciaires sait bien que les prévenus cherchent à décliner les faux semblants d'une identité trompeuse. Mais l'enquête globale et le croisement des sources permettent d'identifier le réseau sous-jacent des liens familiaux tissés par ces femmes apparement anonymes présentant devant leurs juges une défense angoissée en plaidant le veuvage avec une désolante régularité.

\section{Patronage seigneurial et descendance légitime}

II faut bien que cette puissante conscience de soi ait eu pour origine une solide construction familiale. Dès le XVlème siècle, l'état militaire des Bohémiens s'est doublé d'une construction généalogique par le biais de la faveur nobiliaire.

En effet, la protection seigneuriale s'est traduite, surtout au XVlème siècle et dans la première moitié du XVIlème siècle, par un patronage puissant et convaincu des grandes familles ou aussi des lignages plus modestes de la "noblesse seconde". Mais, non contents de protéger systématiquement, - au grand dam des communautés rurales -, une compagnie agitée, insolente et assez prédatrice, les seigneurs portaient sur les fonts baptismaux les "bohémillons", selon la jolie expression employée par Scarron dans le Roman comique pour qualifier les enfants nés dans la compagnie.

Où nous retrouvons nos La Fleur! Le 9 janvier, à Entrains, dans les pays de la Loire de Roger, le baptême de Roger fils de Louis de La Fleur et de Marie Panesin, "bohémiens de nation" eut lieu en présence de son parrain, Roger, duc de Bellegarde, et de sa marraine Anne Marie de Bellegarde, nièce du duc ${ }^{13}$. On peut retrouver la descendance de Jacques Aubert à Saint-Verain, le 23 février 1632:

"Ce jourd'huy vingt troisiesme jour de febvrier l'an mil six cent trente deux, par moy soubsigné curé de Sainct-Verain-des-Boys, a esté solennellement baptisé sur les fonts de l'église dudict lieu Sara, fille de Charles de La Tour et de Marie Reinette, égyptiens de la compaignie du Capitaine Jacques Hauber, de laquelle a esté parrin honorable homme Me Denis Doudeau, procureur fiscal dudict sainct-Verain, et la maraine dame Catherine Raymond, femme d'honorable homme Me jacques Poillet, lieutenant dudct lieu, faict les an et jour que dessus. (signé:) Catherine Remond; Doudeau; P. Naulin (le curé)."'14 
Enfin, pour un dernier exemple, il est assez plaisant de trouver au hasard d'une mention sur un registre paroissial le cas de la jeune Joanne, fille du capitaine Charles de la Rivière, baptisée à Provins le 5 septembre 1653, ayant pour parrain le prévôt des maréchaux de Brie et de Champagne et pour marraine la fille d'un premier président au présidial de cette même ville. ${ }^{15} \mathrm{Ces}$ magistrats avaient tout particulièrement en charge la poursuite des Bohémiens.

L'usage systématique du parrainage eut alors un double effet très structurant. II renforça sans doute les liens de fidélité entre seigneurs et capitaines de bohémiens, mais surtout il donna une légimité par l'enregistrement paroissial à la descendance directe des capitaines de bohémiens et de leurs lieutenants en les constituant en dynasties. ${ }^{16}$ Assurée d'une identité généalogique par l'ancrage dans la parenté nucléaire mise en place à partir du XVlème siècle dans l'Occident moderne, ce qui n'aurait pu être qu'une assemblée de soudards motivés par l'appât des dividendes de l'Etat de guerre se transforma en un réseau de familles stables reliées par des liens collatéraux.

Loin d'être le signe de la marginalité et la conséquence unique d'une répression accrue, la mobilité a favorisé, accompagné en quelque sorte, une manière de construire ce puissant processus de conscience familiale.

En principe, la dissolution des liens familaux est la menace la plus lourde qui pèse sur le monde des pauvres exclus pour une raison ou pour une autre de leur réseau de contraintes seigneuriales ou de solidarités villageoises. Le gibier habituel de la maréchaussée est formé de pauvres hères, de passantes de grands chemins, cherchant solitairement leur pain. II n'en est pas de même des Bohémiens qui peuvent jusqu'à la fin du XVIlème siècle, s'établir avec leurs familles et leurs chevaux pour une halte commode aux abords d'un château accueillant. Le seigneur du lieu brave avec arrogance les ordres royaux et les jérémiades parlementaires le menaçant de lui retirer ses justices au nom de la défense de la "tranquillité publique". Sans doute les derniers feux d'un "devoir de révolte" ${ }^{17}$ bientôt subjugé par le développement de la monarchie administrative...

Voici donc la nature des métamorphoses essentielles des Tsiganes à l'époque moderne. Leur maintien d'une identité collective forte ne viendrait pas d'une mémoire culturelle des origines indiennes, berceau pourtant reconnu de la langue parlée par ces groupes, mais de la double inscription, militaire et familiale, dans une conjoncture européenne paradoxalement "heureuse" de troubles de guerre, de protection seigneuriale et de stabilisation de l'état civil par les décisions royales d'enregistrement paroissial régulier et systématique des enfants baptisés.

Mais le niveau d'analyse ne fait que repousser l'interrogation sur la "grammaire cachée" du processus de construction identitaire d'une minorité.

Comment parvenir en effet par une "anthropologie des profondeurs" à cerner les liens profonds qui relient à travers de multiples métamorphoses les Tsiganes de tous les temps et de tous les pays. La nature de nos sources et nos compétences ne permettent pas de démontrer que l'inscription occidentale a rencontré les habitudes indo-européennes lointaines. Ce serait pourtant 
suivre cette piste fort délicate qui nous attirerait le plus.

\section{Disqualification savante et identité flottante}

Cette construction assura donc aux familles bohémiennes une puissance de survie en dépit des avatars de la persécution. ${ }^{18}$ Mais elle n'eut pas l'heur de plaire au triptyque savant de l'Europe classique. Juristes, clercs et érudits n'accordèrent aux Bohémiens qu'une sorte d'identité flottante, ne leur concédant ni d'être une nation, des pèlerins assidus, des guerriers valeureux, mais une engeance spéciale de vagabonds et de "mauvais pauvres."

En fait, ne convient-il pas d'accorder une importance relative à la description de stéréotypes que chacun peut imaginer sans effort tant ces constructions ne sont solidifiés à travers les siècles? Le paradigme des Tsiganes selon l'expression de Léonardo Piasere ${ }^{19}$ a reposé sur le déni: déni des origines toujours controversées, déni de l'existence d'une nation au sens ancien du terme, déni d'une occupation honnête.

L'évolution de l'image des bohémiens a suivi le contraste de vocabulaire que permet le recours aux textes antérieurs. Là où la tradition employait les termes de "mirabilia", de "merveilles", un Thevet, au XVlème siècle, use du terme de singularité. ${ }^{20}$ Le changement de vocabulaire est significatif d'un renversement d'opinion. A l'étonnement, crédule peut-être, des premières chroniques se subsituait l'observation sceptique des particularismes. ${ }^{21}$

Mais, là encore, la description proposée par les érudits des Bohémiens ne leur donne aucun contour anthropologique défini. Par exemple, Jakob Thomasius, professeur à Leipzig, dans la Dissertatio philosophica de Cingaris, parue en latin en 1671 et en allemand en 1702, illustrait avec une certaine logique le renversement d'attitudes mentales. ${ }^{22}$ L'auteur soutenait que les ducs et comtes de Petite Egypte qui apparurent en Europe à la fin du XVème siècle étaient d'authentiques descendants de princes égyptiens. Mais ceux-ci quittèrent l'Europe et leur place fut prise par des bandes de malandrins qui se moircissaient la peau, se disaient "Egyptiens ou Bohémiens" et vivaient de rapines. Leur art divinatoire n'était que tromperie et il convenait de débarrasser les royaumes de cette engeance disséminée et malfaisante. Camerarius affirmait pour sa part: ${ }^{23}$ "On void et conoit en Alemagne et en d'autres pays certaine sorte de gens ramassez, vagabonds et inutiles, que les Alemans appellent Zigeuner; en Italie on les nomme Cingari, en Espagne Gittani, en France Egyptiens et Bohemiens; Se paissans de l'autrui, n'ayans pire logis que le leur, et rodans de pays en pays."

Ainsi l'opinion savante, soucieuse de ne rien céder du principe d'ordre social qu'elle appellait de ses voeux et nommait la "tranquillité publique", substitua progressivement à la reconnaisance d'une singularité ethnographique, même perçue comme radicalement étrangère, la dénonciation des Bohémiens comme membres d'une contre-société délictueuse, composée de toutes sortes d'individus mal intentionnés et dissimulateurs. 
Celui qui osait manifester un intérêt pour l'étude des Tsiganes devait d'abord s'excuser de traiter un tel sujet. Ce fut pourtant le même Thomasius qui contribua à éveiller la curiosité des linguistes en affirmant que les Tsiganes parlaient une langue qui leur était propre et qu'il ne fallait pas confondre avec un jargon comme le Rotwelch.

A l'époque des Lumières, la confirmation de la parenté de la langue "romani" avec les prestigieuses langues indiennes ne releva nullement le crédit ethnographique des Bohémiens. Le baron Jean-Nicolas-Etienne de Bock, Lieutenant des maréchaux à Thionville, voyageur et correspondant du Journal Encyclopédique, fournit en 1787 une traduction ou plutôt une adaptation de Grellmann, un savant allemand, auteur d'une Histoire des Bohémiens, qui résumait l'opinion du temps. Les Tsiganes étaient d'origine indienne mais ils descendaient, selon lui, de la caste inférieure des Sudders, et conviant Hérodote (lib. III, page 202), il rappelait que celui-ci "fournit la preuve que les Sudders ne sont pas moins adonnés à l'anthropogaphie que les Bohémiens." ${ }^{24}$ A qui eut osé jeter quelque doute sur la valeur d'un témoignage aussi ancien, l'auteur opposait ce principe: "Quelqu'ancien que soit son témoignage, il ne laisse pas d'être d'un grand poids, les moeurs et les coutumes, dans ces contrés éloignées, ne changeant jamais." Les auteurs des Lumières ne voyaient dans les Bohémiens aucune civilité et ne leur concédaient qu'une puissance animale à survivre dans les pires conditions.

II ne reste pas moins que les représentations savantes de "l'autre" aussi dévalorisantes soientelles n'entretiennent que leur propre production en une antienne méprisante sans doute, mais cloturée sur elle-même par le jeu des citations et des références. Une vision à l'évidence réductrice d'un sujet marginal.

Si les Tsiganes n'avaient été qu'un ramassis de soudards battant la campagne, la société de persécutions ne leur aurait pas accordé la moindre chance de survie collective. L'histoire de l'Europe est pleine de peuples ou de groupes à la renommée éteinte dont les noms ne sont même pas parvenus jusqu'à nous!

Contrairement à la théorie produite par ceux que j'appellerai les "esthètes de la dissidence", la production du discours d'exclusion dans la pensée occidentale n'a pas toujours été cohérent et continu. II a pu se construire - c'est du moins ce j'observe pour mon objet d'étude - comme par défaut, expression intermittente d'une attention flottante de l'opinion savante, selon des angles de vue très variables. Ces discours ont produit des images partielles donnant au sujets incriminés une sorte d'existence floue, sans consistance, une sorte d'insignifiance vitale qui pouvait assurément devenir plus dangereuse dans certaines conjonctures que tous les refus organisés.

II nous reste donc à revenir sur l'obligation qui nous est faite de confrontation avec les conjonctures.

Je ne nie pas qu'il existe des conjonctures complexes de passage à l'acte, produit d'une alchimie singulière hic et nunc qui provoque le basculement brutal des personnes physiques et non 
d'âmes éthérées dans l'exclusion, plaçant désormais la victime et son bourreau de part et d'autre d'une ligne de démarcation; mais j'affirme aussi que la disqualification juridique et intellectuelle d'un groupe humain n'implique pas automatiquement sa liquidation physique, et que l'on peut même fort bien s'accommoder d'être mal aimé.

Ainsi les Bohémiens ne souciaient bien peu de la renommée désavantageuse que leur manière de vivre, largement imitée de celle de leurs maîtres, leur conférait auprès des paysans et dans une opinion urbaine en formation; bien au contraire, ils entendaient profiter de la crainte qu'ils inspiraient. Donnons une dernière fois la parole au capitaine La Fleur; au procureur qui l'interroge, le 22 mai 1603, sur les moyens d'existence de la compagnie, il fait cette réponse: "...il déclare en oultre qu'il ne pense estre fort mal faict de prendre ainsy les poulles, veu que les renardz les mangent bien qui sont bestes inraisonnables et qu'à plus forte raison eulx comme creatures raysonnables les doibvent bien manger, d'aultant qu'il fault vivre. Adjouvent encores S'ilq ne vivoient poinct de ce moien et de telle facon qu'on ne les appelleroit des Egyptiens."

En conclusion, nous pensons tenir ici un cas strictement inverse du processus classique d'exclusion dans l'Europe moderne. A l'ordinaire, les autorités judiciaires disposaient des armes d'un discours de dévalorisation cohérent; ${ }^{25}$ elles s'acharnaient à compromettre les prévenus se débattant dans le cadre terrifiant d'un procès personnel pour briser par la segmentation des affirmations et des témoignages leur défense fragile et obtenir les aveux indispensables.

En d'autres termes l'État moderne français, pourtant l'un des plus accomplis par la mise en oeuvre de l'absolutisme et de la "maxime de l'ordre", ne porte pas en soi un processus d'exclusion univoque.

Tout dépendait de l'agencement des positions imposées par des conjonctures mentales complexes aux tenants de la normalisation, et à leurs cibles. 
${ }_{1}^{1}$ François de Vaux de Foletier, Les Tsiganes dans l'Ancienne France. Paris: Connaissance du monde, 1961; Henriette Asséo, Les Tsiganes. Une destinée européenne. Paris: Gallimard-Découvertes, 1994.

${ }^{2}$ A. D. Loire-Atlantique , B 27, Fol 31 Vo.

${ }^{3}$ A. D. Loire-Atlantique, B 17, Fol 59.

${ }^{4}$ D'après la copie publiée dans Adolphe Lecocq, "Bohémiens à Chartres en 1571", in Lettres Beauceronnes, recueil de 45 pages, Chartres, 1865, pp. 214-234. Communication de M. Couturier, secrétaire de la Société Archéologique d'Eure-et-Loir. L'original se trouvait chez Me Besnard, notaire à Chartres, 20 rue du Cheval Blanc.

\section{“Le Jeudy dixieme jour de may 1571}

Aujourdhuy, dacte de ces presentes, en presence du notaire et des tesmoings soussignés, est comparu Nicolas Lescuyer, natif de la Petite Egipte, cappitaine et conducteur de trois mesnages de sa compaignie, certiffie que François Aubert, aussi naptipf de la Petite Egipte, aesté soubz sa charge avec sa femme, sa mère, famille et serviteurs, depuis le temps qu'il faict partie d'icelle Petite Egipte jusqu'à cejourdhuy, et qu'il s'est toujours bien porté et contenu partout où il a passé, suivant le pouvoir audict capitaine donné par le Roy et ainsi quil est contenu par ces lettres patentes qu il a présentement exibez, portant sauf garde et sauf conduict, donnez à Paris le dixieme jour de décembre, l'an mil VcLXIX; ainsi signé Descoulay, et scellées du grand scel de cire jaulne, pour lesquelles luy est permis passer par tout le royaulme de France en bonne seureté avec lesdictz trois mesnages, sans les molester en aulcune chose, et deffences à tous les juges, Prevts des Marchaulx, maistres des Gallères et aultres, ne leur donner aulcun empeschement, mais au contraire leur donner confort et aide. Lequel Lescuyer, capitaine susdict, a, en présence dudict notaire et des tesmoings cy après nommez, donné congé audict François Aulbert qui lui a requis et demandé, de s'en aller et départir hors de sa compaignie avec sa femme, famille et serviteurs pour chercher ses frères, en quelque lieu qui les pourra trouver, et ce faisans luy a donné pareille puissance que a, par ces dictes lettres-patentes, qui est de ne mesdire audict François Aubert, sa femme et famille; et deffenz à tous juges, Prevost de Marchaulx, Maistres des Gallères, que aultres, ne leur donner aulcun empeschement. Ains de leur donner confort et aide et les laisser passer par tout le royaulme de France sans leur faire aulcun tort, tant à leur personne que biens et par les lieux où ils passeront, leur donner logis pour ung jour ou deux par villes, villaiges ou bourgades de ce dict royaulme en payant leurs despens raisonnablement. Ce que moy notaire susdict, certiffie les dictes lettres porter icelle puissance. Dont acte, auquel estoient presens Françoys Cerceau et Laurent Delaborde, demeurans à Chartres, lesquelles parties ont déclarés et lesditz tesmoings ont signés.

Cerceau Delaborde de Baignaulx notaire"

${ }^{5}$ Et l'on comprend le rôle essentiel des parrainages pour garantir des fidélités sans doute fluctuantes mais maintenues dans une constellation d'alliances précises délimitées par la religion.

${ }^{6}$ A.D. Moselle, B 4577, procès criminel instruit devant les échevins de la ville et prévôté de Thionville, mai 1602. Les pouvoirs des juridictions semblent assez complexes. En effet le gouverneur dépendait au moment du procès des Habsbourg.

C'est pourtant sur une ordonnance "Par le roy" donnée à Bruxelles le 2 septembre 1560 "a noz amez et feaulx les Gouverneurs, President et gens de notre Conseil à Luxembourg" que s'appuie la demande des gouverneurs de la province par lettre du 28 avril 1603 à leur "Très cher et bien aimé confrère" le comte de Wiltz. 
${ }^{7}$ François de Vaux de Foletier a découvert ce dossier.

${ }^{8}$ A.D. Moselle, B 1577. En 1601, un égyptien de la compagnie du capitaine la Fleur nommé Guillaume La Pointe, surprenant sa femme avec un autre égyptien, tua l'épouse et le rival.

${ }^{9} \mathrm{Ce}$ jeune garçon pourrait bien être le neveu du capitaine La Fleur.

${ }^{10}$ La législation est analysée dans Henriette Asséo, "Le traitement administratif des Bohémiens" dans Henriette Asséo et Jean-Pierre Vittu, Problèmes socio-culturels en France au XVIle siècle. Paris: Klincksieck, 1974, pp. 9-88.

${ }^{11}$ A.D. Aisne, bailliage de Vermandois, 89 2. Cf., Amedee Combier, Etude sur le baillage de Vermandois et siège présidial de Laon. Paris: E. Leroux, 1874, p. 334.

${ }^{12}$ A. D. Indre-et-Loire, maréchaussée de Tours, liasse, année 1728-1729.

${ }^{13}$ Registre paroissial d'Entrains dans la Nièvre, baptême du 9 janvier 1642, communication du conservateur des archives de la Nièvre.

${ }^{14}$ Texte intégral extrait des registres paroissiaux de Saint-Verain, dans la Nièvre, également communiqué par le conservateur des archives de la Nièvre.

${ }^{15}$ A. D. Val-de-Marne, Provins, Saint-Quiriace, collection communale, GG 85, 1653-1662.

${ }^{16}$ Herman Gabriel, "Le parrainage, "I'hospitalité" et l'expansion du christiannisme", Annales H.S.S., 52 (1997), pp. 1305-1338 montre comment le parrainage ne joue pas sur la transmission des biens comme l'adoption mais sur la consolidation du nom. II parle en p. 1331 du parrainage chez les Tsiganes contemporains.

${ }^{17}$ Arlette Jouanna, Le devoir de révolte, La noblesse française et la gestation de l'Etat moderne, 1559-1661. Paris: Fayard, 1989.

${ }^{18}$ II est surprenant de voir que l'intériorisation inconsciente de cette tradition généalogique se traduit par la dinstinction subtile entre les "bonnes familles" c'est-à-dire "vieilles familles" et les nouveaux-venus fort mal vus par ces dernières alors que la société extérieure des "gadge" met tout ce monde dans le même sac. cf. Norbert Elias, John L. Scoton, Logiques de l'exclusion. Paris: Fayard, 1997.

${ }^{19}$ Leonardo Piasere, "De origine Ciganorum" dans Paul-Henri Stahl (éd.), Etudes et documents balkaniques et méditerranéens. 14, pp. 105-126.

${ }^{20}$ La Cosmographie universelle d'André Thevet, ...illustrée de diverses figures des choses plus remarquables veüs par l'auteur... Paris: P. L'Huillier, 1575, 4 tomes en 2 vol. in-Fol, tome 2, p. 916 verso.

${ }^{21}$ Communication de Michel Mollat du Jourdin, "L'altérité, découverte des découvertes", dans Jean Ceard et Jean-Claude Margolin (éds), Voyager à la Renaissance. Maisonneuve et Larose, 1987, pp. 305- 318, p. 306 (sous la direction de Jean Ceard et Jean-Claude Margolin).

${ }^{22}$ Jacob Thomasius, Dissertatio philosophica de Cingaris, in disputationem proposita Lipsiae a M. Jacobo Thomasio, ... respondente Johanne Christophoro Schmid, ... anno 1652, 3. idus septembris... Nunc recusa anno 1671. Lipsiae: literis J. E. Hahnii, 1677, in - 40, p. 24.

${ }^{23}$ Bibliothèque Mazarine, ms 18790, Les Méditations philosophiques de M. Philippe Camerarius, docte jurisconsulte et Conseillier au Senat de Nuremberg ville impériale... en trois volumes, trad. par S. G. S., 
(Simon Goulard, de Senlis), MDCX, à Lion, vol. 1, Pour la veuve d'Antoine de Harsy, Livre 1, chapitre XVII Des Aegyptiens, ou Bohemiens, p. 85. II s'agit du 3ème volume datant de 1610 d'un ensemble qui en contenait déjà deux autres.

${ }^{24}$ Jean-Nicolas-Etienne baron de Bock, Recherches historiques sur le peuple nomade.appelé en France Bohémien, et en Allemagne Zigeuner: avec un vocabulaire comparatif des langues Indienne et Bohémienne, traduites de l'allemand de M. Grellmann par M. le B. de B... Paris et Metz, 1787, pp. 91, 48.

${ }^{25}$ Même s'il était le résultat d'une culture de compromis comme l'a monté Carlo Ginsburg à propos du sabbat des sorcières. 